\title{
Exploration of Applying Teaching Methodology to Massage Manipulation Liangbing Yang ${ }^{1}$, Ying Shao ${ }^{2}$, Haikuan Wang ${ }^{3}$ \\ ${ }^{1,2,3}$ Guangzhou University of Chinese Medicine, Guangzhou Guangdong, 510006, China
}

\author{
Keyword: Massage Manipulation, Classroom Teaching, Teaching Methodology, Staged Teaching \\ Method.
}

\begin{abstract}
During recent years, with the increase of material living standard, people have paid more and more attention to the physical conditions. Thus the massage manipulation teaching which can impose certain impact on people's health has also gradually attracted wide attention. Especially during the 10- year integral moving of Guangzhou Higher Education Mega Center, our university has developed the Massage Manipulation - interscholastic course of Guangzhou Higher Education Mega Center. To give the lecture well among those students having no medical foundation and let them actually master the essentials, further to apply the knowledge to daily life and safeguard health, it requires students to possess skillful massage manipulation. Therefore, teaching of massage manipulation has just become the key teaching content. On the basis of briefly illustrating the countermeasures of promoting teaching quality of massage manipulation, it takes the staged teaching method as an example and analyzes the concrete method of applying the teaching method to massage manipulation, with the hope of offering corresponding support and reference for the development of massage manipulation teaching.
\end{abstract}

\section{Introduction}

Massage manipulation is one essence of traditional Chinese medicine. Accompanied by human's advocation to return to nature and enthusiasm for naturopathy, massage manipulation has become the first choice for people to prevent disease and keep fit, and also adjust the sub-health. On the basis of making concrete analysis on the massage manipulation teaching, it puts forward the countermeasures to improve the teaching quality and apply relevant teaching methodologies to practice, with the hope of creating conditions for the orderly development of teaching activities.

\section{Method to Fatherly Improve the Massage Manipulation Teaching Quality in the New Period}

\section{Positively Introducing Several Teaching Methods, Letting Students Learn Easily}

Students of massage manipulation teaching are from the other 9 universities of Guangzhou Higher Education Mega Center, who are not majored in Medicine. Having no medical foundations, they are quite different from each other, whose learning abilities vary from each other as well. Within the limited time, it is hard for students to learn the teaching contents. Therefore, in the teaching practice, we have tried to introduce many teaching methods, such as staged teaching method, mechanics-centered teaching method, comparative teaching method and PBL teaching method. Under the reasonable guidance of several teaching methods, students can complete the learning of massage manipulation courses; further to fatherly improve the learning effects ${ }^{[1]}$. By taking PBL teaching method as an example, it usually takes the introduction of question as the foundation. Combined with the questions assigned by the teacher before class, students can search and sort materials, formulate feasible learning methods in classroom discussion and also make learning summary together with teachers. In this way, under the guidance of teachers, students can complete 
the exploration of relevant knowledge, who will absolutely have deeper understandings about the teaching contents and certainly impose positive impacts on the reinforcement of teaching effects.

\section{Creating Easy and Free Classroom Learning Atmosphere}

According to relevant investigations, in a comparatively easy and free learning atmosphere, learning abilities of students can be motivated to the hilt, which is beneficial for the father reinforcement of learning effects. On that basis, we have explained all knowledge of the massage manipulation in the lab. Classes are quite interactive for students to operate positively, so as to create comparatively easy and free classroom teaching atmosphere invisibly. For another example, while teaching the head nursing by massage manipulation, in order to create comparatively easy learning atmosphere, we have played soft music in class particularly, let students keep comparatively easy mind while learning the massage manipulation, strengthened their understandings about the knowledge and fatherly improve the teaching effects of classroom.

\section{Strengthening the Basic Training}

While learning the massage manipulation, students are required to master proficient skills and certain strength and physical fitness. Especially for girls, because of their weaker strength of muscles and insufficient stamina, it is hard for them to carry out massage manipulation persistently. Once they cannot obtain corresponding learning effects after a long time, their learning interests will gradually become weakened, which is bad for the effective development of massage manipulation course. Therefore, in the early period of learning, we teach students to learn some flexible and soft techniques, which do not need too much strength. Such as the thenar eminence massage, we let students to be interested in the technique and produce the sense of achievement after mastering the technique, so as to lay a foundation for learning other techniques in the future. After that, we will fatherly emphasize on practicing basic training. Only by guaranteeing the proficiency of basic training, can they offer corresponding guarantees for obtaining learning effects later, so as to eventually realize the realm of couple hardness with softness.

\section{Implementing Self-protection Education on Students}

In the early period of teaching massage manipulation, because students are lack of correct understandings about the massage manipulation teaching, they have insufficient consciousness of self-protection, thus it is quite hard for them to operate in a correct way. It is quite easy for students to have stubby fingers and deformed joints. In severe cases, they may suffer soft tissue inflammation ${ }^{[3]}$. Therefore, we should analyze attention points of every movement in teaching process, and guide students how to protect themselves while carrying out massage manipulation, further to adjust their physical conditions in a scientific way. In the meanwhile of guaranteeing the health of students, we can promote the massage manipulation to obtain obvious improvement. For instance, while students are learning to press lower back with palms, we especially emphasize that students should stand on the ground by forefoot and exert force on the lower backs of patients with the help of their own weights. In this way, students can easily complete the massage manipulation and patients can feel even strength and comfortable sensation.

\section{Applying Staged Teaching Method to Massage Manipulation Teaching}

To reasonably apply the staged teaching method to the massage manipulation, it simply means to divide the massage manipulation teaching into stages. Based on different stages, students can complete the learning about relevant knowledge step by step, so as to obtain good learning effects. Next, we will make concrete analysis on the application of staged teaching method.

\section{Stage of Imitating Essentials of Movements}

In this stage, students have still just learned the massage manipulation, thus they can merely imitate the movements of teachers. Specifically, there are three teaching links as follows: 
Firstly, it is the interpretation of massage manipulation theory. While we are interpreting the theories of massage manipulation, combined with the learning characteristics and requirements of students selecting the interscholastic course of Guangzhou Higher Education Mega Center, we try to use vivid languages to interpret the concept and basic essentials of massage manipulation. If we talk about the swaying techniques, we emphasize that the subjective swing of forearm just looks like the motion of a clock, which needs a fulcrum. Students can clearly understand the essentials and patterns of essentials. During the process, we can also properly insert the comparative teaching method. By comparing different technical movements, students can have deeper understandings about the massage manipulation, so as to lay a foundation for the learning in future.

Secondly, it is the performance demonstration. Performance demonstration for students can be interwoven with the interpretation of theories. We can also require students to imitate purely. In addition, we especially attach importance to standard and proficient movements in the actual interpreting process, and also stimulate their leaning interests by interesting demonstrations. Because we give the lessons in the lab, we can demonstrate the manipulation for students directly and explain the requirements of the technique simultaneously. What's deserves attention is that, as those quite difficult movements, we emphasize on demonstrating movements after decomposing them into parts, highlight the key points of movements, which is convenient for students to imitate and practice.

Thirdly, it is the practice of manipulation. To actually master the massage manipulation in the learning process, they should practice it repeatedly for a quite long time. Especially those complicated movements with quite difficult techniques, students should consciously train themselves for a long time. Meanwhile, while students are practicing the massage manipulation, we should observe every student and immediately give advice for their learning difficulties. Besides, we should correct those non-standard operations during the practicing process, so as to guarantee students can actually master the massage manipulation after training for a long time and possess the qualification for clinical preparation ${ }^{[4]}$. In the stage of clinical preparation, students can initially apply their massage manipulation to human bodies by practicing on human bodies, further to deeply understand different parts of human bodies in practical application process, strengthen the proficiency of all massage manipulation techniques, guarantee that they can proficiently transform different movements in practical massage manipulation, and also possess the basic abilities of health-care massage manipulation.

In the stage of imitating essentials of movements, with the help of theoretical interpretation, movement performance and technique practicing, students can basically master the essentials of massage manipulation and form rigorous learning attitude. Thinking, coordinating and observing abilities of students can be properly exercised, which is good for students to learn in the next stage.

\section{Preparatory Stage of Linking Theory with Practice}

It is the later period when students to practice massage manipulation in classroom. During this stage, most students can master corresponding training skills and practice the massage manipulation. Therefore, they begin to link theory with practice and hope their massage manipulation can be actually applied to patients, so as to verify the curative effects of health-care massage manipulation. Based on that, in order to fulfill students' wishes for linking theory with practice, let learning in the practical stage can obtain good effects, teachers can start to guide students to preliminarily link the theory with practice, so as to reinforce the massage manipulation exercise.

Firstly, it should strengthen the massage manipulation on human bodies. After completing the interpretation of massage manipulation theories, teachers should organize students to strengthen their practices on human bodies. According to different parts of human bodies, teachers can train students on purpose. Moreover, according to the ages, basic physical conditions and situations of different parts of human bodies, they can arrange reasonable massage manipulation exercise, guarantee the movements and strength can satisfy the demands of training object, so as to make preparations for the learning later. 
Secondly, according to the learning abilities of students, teachers can organize corresponding service teams and offer health-care massage manipulation for teachers and students. Targeted at concrete objects, students can reinforce their massage manipulation; understand the conditions of sub-healthy population and massage manipulation skills they should possess, further to actually promote massage manipulation of students to be correspondingly strengthened ${ }^{[5]}$. Setting of such a teaching method can eliminate the school-weariness and psychological fatigue during repeated reinforced training, and also let students to produce certain spirit of service and sense of achievement after obtaining service, further to motivate the learning enthusiasm of students to the maximum. Besides, targeted at the team of service, teachers can also properly lead students to offer social services in squares and nursing homes. In the meanwhile of offering public services for the public, students can also properly exercise their massage manipulation.

Lastly, we can organize students to participate in the professional social practice in vacations, so as to strengthen their massage manipulations. Based on the development of that teaching link, teachers should give lectures to students before the vacations, teach medical consultation theory, techniques and therapies for common diseases to the students and make demonstrations. On the basis of improving students' cognition and understandings about the knowledge, teachers can organize students to join in more professional social practice and make preparations for letting them link theory with practice. For instance, students can be organized to practice in massage manipulation department of relevant hospitals, or carry out massage manipulation on relatives or family members who suffer diseases. During the process, students should immediately ask teachers for advice in case of uncertain conditions. They must avoid blind therapies, in case of resulting in serious consequence. After the opening of school, teachers should also organize students to reflect on and summarize social practices, let them share their experiences of joining in professional social practices, so as to lay a foundation for learning in future. In this way, students cannot only actually participate in the massage manipulation practice, but also obviously improve their learning enthusiasm.

\section{Stage of Linking Theory with Practice}

During the teaching of health-care massage manipulation, the stage of linking theory with practice specifically refers to that, students should complete the practice of massage manipulation, practice on different healthy human bodies and patients. During this period, teachers should require students to distinguish the differences and similarities between exercise on regional part and disease treatment, and also deepen their understandings about the effects of massage manipulation, guarantee students can select rational massage manipulation by actually combing with illness in practical operation, further to obtain the best learning effects.

\section{Conclusion}

Above all, health-care massage manipulation teaching has exerted extremely importance significance on the development of massage manipulation courses. Only by attaching great importance to the teaching practice and scientifically guiding students, and also introducing advanced teaching methods, can teachers satisfy learning requirements of students. By strengthening the connection between theory and practice, they can cultivate the practicing abilities of students, so as to lay a solid foundation for students to master the massage manipulation techniques and development in future.

\section{Acknowledgement}

Foundation Program: Innovative University-strengthening Engineering, Researches based on the Resource Construction of Interscholastic Course of Guangzhou Higher Education Mega CenterMassage Manipulation Researches on Optimized Teaching Mode of Interscholastic Course of Guangzhou Higher Education Mega Center-Massage Manipulation. 


\section{References}

[1] Zhang Nengzhong. Massage Manipulation Teaching and Practicing, Chinese Manipulation and Rehabilitation Medicine, 2015(2):49-49.

[2] Sun Xiao, Li Jing. Progress of Applying Motion Learning and Control Theory to Traditional Chinese Manipulation, Chinese Journal of Rehabilitation Medicine, 2013,28(1):90-92.

[3] Zhang Xiaowen. Several Thoughts on Reforming Practicing Lessons of Massage Manipulation, Value Engineering, 2013(27):223-224.

[4] Lei Longming. Improvement of One-finger Scrubbing Teaching Method in Massage Manipulation and Teaching Experiences, Journal of Guangxi University of Chinese Medicine, 2015,18(3):95-96. 